\title{
RADIOMICS BASED BREAST MALIGNANCY INDEX TO DIFFERENTIATE PATHOLOGICAL CHANGES DUE TO NEOADJUVANT CHEMOTHERAPY
}

\author{
Priscilla Dinkar Moyya ${ }^{1}$, Mythili Asaithambi ${ }^{1}$, and Anandh Kilpattu Ramaniharan ${ }^{2}$ \\ ${ }^{1}$ Vellore Institute of Technology, Vellore-632014, Tamil Nadu, India \\ ${ }^{2}$ University of Alabama at Birmingham, Birmingham-35233, Alabama, United States
}

Corresponding Author: Mythili Asaithambi

Email: mythili.asaithambi@vit.ac.in

https://doi.org/10.34107/BiomedSciInstrum.57.04219

\begin{abstract}
The leading cause of deaths among women in the world is Breast Cancer. Neoadjuvant chemotherapy (NAC) offers effective treatment results, thus reducing tumor aggression and allowing treatment monitoring. The Dynamic Contrast Enhanced (DCE) MRI plays a vital role in assessing the treatment response due to NAC. However, quantifying the treatment response in low-grade tumours is visually challenging. Radiomics is an evolving field of medical imaging that reflects the histopathological variations in breast tissues. Integrating radiomics with breast DCE-MRI provides clinically useful measures in evaluating the NAC response. In this work, we have formulated an index called Radiomics based Breast Malignancy Index (RBMI) using texture and Haar wavelets to differentiate the radiological differences of breast tissue due to NAC. The statistically significant radiomic features extracted from 20 DCE-MR images obtained using TCIA database were used in the calculation of RBMI. Results show that, RBMI could statistically differentiate $(\mathrm{p}=0.007)$ the treatment response between visit-1 \& 2 due to NAC with mean and standard deviation values of $334706.5949 \pm 93952.5123$ and $296354.9720 \pm$ 77120.6718 respectively. Hence, RBMI seems to be a clinically adjunct measure in evaluating the treatment response of breast cancer due to NAC.
\end{abstract}

Keywords: Breast Cancer, DCE MRI, Neoadjuvant Chemotherapy, Radiomics, RBMI

\section{INTRODUCTION}

The foremost public health problem in worldwide is cancer and the second leading cause of death is breast cancer [1]. In 2016, The Global burden of diseases, injuries and risk factor study assessed the number of breast carcinoma cases in India as 1,18,000 and reported that these incidents got increased by $39.1 \%$ in 26 years [2]. Early discovery and classification of breast cancer helps to improve the therapeutic treatments to patients.

The widespread methods used for breast cancer analysis is mammography and ultrasound that shows a prominent role in medical practice. The ionization radiation of mammography and perceiving contra lateral malevolent tumors in ultrasound are principal drawbacks. The mammography is not acceptable for high dense breast women and poses challenges in the detection of lesions due to the absence of functional information. Magnetic Resonance Imaging (MRI) considerably enhances diagnostic procedure and is characterized by its high sensitivity in detecting tumors which has greater potential for diagnosing cancer, early detection, and therapeutic effects with minimum invasiveness, taking in to account that MRI fails in dense breast tissues [3]. Therefore, Dynamic Contrast Enhanced Magnetic Resonance Imaging (DCE-MRI) of the breast is very sensitive in early stages of breast cancer finding and suitable for dense breasts. It takes the entire breast volume as slices and measures the information interpreting the breast vascularity [4]. This modality mainly provides morphological, dynamic and offers the potential for numerous and quantifiable perfusion and microvascular parameters. The lesion recognition sensitivity is nearly $100 \%$ for breast DCE-MR imaging and specificity is low, with recorded rates ranging from 30 to $70 \%$ [5]. 